\title{
Research on the Novel Teaching Model of Translation Workshop Based on Hadoop Platform
}

\author{
Na Zhai \\ Translation Department, Xi'an Fanyi University, 710105 Xi'an, China \\ zhaina999@163.com
}

\begin{abstract}
Keywords: Hadoop platform; translating technology; translation workshop
Abstract. The traditional translation teaching mode pays little attention to students' practical and cooperative abilities, not to mention the translating technology required by the occupation at present, which has become a big challenge for the cultivation of translation talents meeting the social needs. Under this circumstance, the author puts forward a novel teaching model of translation workshop based on Hadoop platform, which can combine the teaching of traditional translation techniques and the application of modern technology. Keeping up with times and the occupational development, the teaching model is worth popularizing.
\end{abstract}

\section{Introduction}

In 2007, Google first proposed the concept of cloud computing. Then, IBM launched the "blue cloud" program and broke this concept into market successfully. IBM also defined cloud computing in its technological white paper: "cloud computing is a systemic platform or a type of application which can provide services of provision, configuration, reconfiguration and deprovision according to the customers' needs" [1]. Cloud computing is a kind of delivery and application mode of IT resources, i.e. to get the needed hardware, platform, software and service resources through the network (including the Internet and intranet) in a required or scalable way [2].

Cloud computing can provide access to network at all times and places. It has the advantages of reliable and safe data storage, user convenience and strong operation ability. Moreover, it can offer on-demand services with low price and high efficiency. Based on the mentioned advantages, cloud computing will change the current working mode and commercial mode fundamentally, which will have deep and significant impact on all walks of life, including the area of translation.

\section{Cloud Computing and Translation}

The contribution of cloud computing made to the field of translation (especially translation technology) is the leaping quality of machine translation. The concept of translation technology includes the information technologies and electronic translation tools used in translation industry, such as machine translation ((MT), translation memory system (TM), term management system, software localization tools, online dictionary and terminology database and corpus [3].

Compared with the manual translation, the advantages of translation technology are mainly reflected in three aspects: terminology unifying, time saving and cost decreasing. At the same time, a large number of projects have also established certain database for translation technology and provided data support for the future translation work. In this context, translation teaching needs to keep up with time and occupation, not only cultivate students' traditional translation skills of language and culture, but also let the students grasp translation technology and practical ability.

\section{The Construction of Hadoop Learning Platform}

The public cloud services launched by large-scaled domestic Internet companies include Baidu open cloud, Ali cloud and Tencent cloud. But they don't provide special cloud platform for education, nor the open source code. It is difficult to make modification, expansion or integration according to the teaching demand. In addition, the learning platform don't allow personalized usage or cluster 
application of software. Therefore, this study researches on the open-source cloud computing system - Hadoop, which is the private cloud translation learning platform built according to the specific requirements for undergraduates of translation majors. Hadoop platform uses Hemlock-the tool packet based on XMPP to realize the instant communication between users and provide the corpus database for the learning platform, adopts OpenMeetings - the video conference system development kit to build functions of video and audio communication, applies Roller Webblogger to establish the message boards and blogs, employs Wiki for collaborative document editing and management and Dropbox to realize the synchronized update of translation memory and terminology databases. By use of the terminal equipment connecting to internet, teachers and students can log on the learning platform whenever and wherever possible for independent or collaborative learning.

The learning platform has five functional areas: Learning Platform Management, Course Information, Cooperation and Interaction, Translation Works Submission and Feedback and Translation Tools. Teachers and students can register an account in the area of Teaching Platform Management, then enter into the software service layer of the learning platform by their entrance for translation teaching and learning. The maintenance officer can enter the service and infrastructure layer of the platform for maintenance and management. The are of Course Information is mainly used for teachers to post information of translation course, translation tasks or materials for students to study and discuss. Through the area of Cooperation and Interaction, the teacher-student or student-student collaboration, cooperation and interaction can be realized: Web instant communication allows the functions such as voice and text sending, offline messages pushing, history message recording and message leaving etc.. The collaborative drawing function of the whiteboard supports the collaborative writing and discussion of teachers and students. Audio and video communication function can be used to hold the translation advance meetings. Translation forum can be used for teachers and students to discuss translation learning. Wiki cooperative translation system can achieve collaborative writing or discussing of the best translation version of a group. The area of Translation Works Submission and Feedback can show students' initial translation works, through which the whole translation learning process can be seen clearly. The best translation works, the model essays, comments and reflection journals can be shown here. The learning platform is embedded with some widely used translation tools at present, such as Trados, Wordfast, CAT and related terminology database, electronic dictionary, search engines and corpus.

\section{The Design of Teaching Model Based on Hadoop Platform}

Translation workshop is a teaching mode focusing on the process of practice, taking students as the center and guided by the talent-cultivating goals. In this teaching model, a group of students engaged in the translation activities will gather together to carry out extensive and heated discussion on a specific translation task to exchange views., negotiate and finally decide on a best version agreed or accepted by all members of the group [4]. The teaching model of translation workshop is a type of seminar based on the translation practice, which encourages students to study translation in translating, in cooperating and in discussing.

The author here puts forward a novel teaching model combining the technological advantages of Hadoop platform and collaborative features of translation workshop. The teaching methods include both in-class interaction and off-class collaboration are adopted to complete the translation teaching. Every two weeks is designed as a learning unit of the translation workshop. Before the opening of the course, the students have been trained with the usage of the learning platform and the work flows of the novel teaching model. The students have been grouped, every member can take turns to be the leader of the group. The author will take the learning unit - The Translation of Medicine Specifications as an example to introduce the details of the new teaching model.

Assignment of Translation Tasks. The teacher should scan the real English medicine specification scan and save it in PDF format, then work out the translation requirements and recommendations: e.g. Students should pay attention to the fixed format, terminologies and style of the medicine specification; Students can search online for the similar bilingual drug instructions for reference. 
Students can download and use the PDF to Word Converter v2.0.0.151 In the learning platform to convert PDF files to word format for easy editing. Teachers issue the translation tasks, requirements and recommendations in the area of Course Information, and push the message to students through the Web instant messaging tools. Students log on the learning platform to get the translation task and participate in the translation advance meetings held by the group leader through the Web instant messaging system for discussing translation materials, screening and analyzing problems and difficulties, developing the project schedule and allocating the task.

The Autonomous and Collaborative Translation Process of the Students. The medicine specification contains too many terminologies. After receiving the translation task, students can first use the online dictionary to search the meaning for accurate understanding and expressing of the specialized vocabularies. Subsequently, they can search in Baidu Library or with academic searching engine - GFsoso for a similar bilingual drug instruction, thus to be familiar with the fixed format, terms and the style for reference. For example, medicine instruction usually include contents such as ingredients, description, indications, suggested dosage and caution etc.. Simple sentences are usually used to introduce the information of the medicine in order to be clear, concise, accurate. Wiki collaborative editing system can be used to establish, edit, supplement the encyclopedic knowledge of translation,thus to realize the knowledge sharing in learning, which is a typical feature of collaborative learning in cloud era. When it's difficult for the students to translate some difficult sentences independently, they can interact with each other to discuss the problems and doubts, try to answer the questions raised by other students, or share their experience, thinking or deep understanding of the translation.

Submission and Peer Assessment of the Translation Task. The students will send their independently finished version to the submission area. Besides the completion of his or her own translation task, each student is also responsible for the evaluation of the other student's translation version, and the comments will be uploaded to the feedback area. Students can choose to modify and improve their translation selectively based on the comments. There may be some difficult translating problems can not be solved independently by the students. The group leader needs to work out a list of these problems or questions for students' further discussion or teacher's resolution before class.

In-class Presentation, Discussion and Summary. The group leader should select the best translation through collective discussion, present it to the class by slides, explain key points of translation, sum up the advantages and disadvantages of the translation version and put forward the unsolved difficult problems for students' discussion and teacher's guidance. During the whole process, the teacher needs to record the key part of students' reporting, explain and give answer to the difficult issues reflected in the question list or report. At last, the teacher needs to give comments to the completion of translation tasks including the quality of translation version, the use of translation technology and collaboration or cooperation between students. After the classroom teaching, the group leader should adjust the translation version based on the students' discussion and teacher's guidance and upload it to the learning platform.

\section{Summary}

The novel teaching model of translation workshop based on Hadoop platform can realize the organic combination of in-class discussion and off-class collaboration of the students and establish a system of knowledge sharing and practical learning. The teacher can provide solution to students' problems in class and supervise students' performance after class to improve their translation ability. The teaching model has gained recognition and support from the students. This shows that the teaching mode can change the current shortage of translation practices of undergraduates. Its application is not only feasible, but also necessary. Of course, the shortcomings of the teaching model can also be seen from the feedback of students: e.g. the limited role of peer's assessment, the students' lack of computer and network knowledge, the higher demand for the teacher(he or she needs to log on the platform, offer uninterrupted supervision and guidance to the students even in his or her spare time). All these will be the directions for further study in the future. The author will make continuous 
improvement to the teaching mode, thus to make contribution to the cultivation of professional translation talents meeting the social needs.

\section{Acknowledgments}

This work was financially supported by the 13th Five-Year Planning Project of Shaanxi Educational Science Institute (SGH16H309).

\section{References}

[1] Michael Mille: Cloud Computing (Machinery Industry Press, Beijing, China 2009).

[2] P. Wang and J.P. Zhang: Modern Edu. Tech. Vol. 11 (2008), p. 81

[3] H. L. Wang: Edu. Theory and Practice. Vol. 6 (2016), pp. 61-62

[4] M. Li and W. H. Zhong: Chin. Trans. Journal. Vol. 4 (2010), p. 25 